\title{
Back to the Future... the Challenge for Cancer Education and Training in Developing Countries
}

\author{
Arthur M. Michalek
}

Published online: 6 July 2013

(C) Springer Science+Business Media New York 2013

It is the best of times and the best of times. It is time for the AACE-CPEN Annual Meeting. This year our family will gather in Seattle to share lessons learned and to plot new adventures. For many of us, this is the highlight of our year where we take time out from our busy schedules to renew acquaintances and make new ones. A time to learn new skills, share knowledge, and become reenergized. This year's meeting promises to be an excellent one. A number of excellent podium and poster presentations have been scheduled which should certainly satisfy our need for new knowledge and skills. In addition, a number of speakers have been invited to share their expertise. Dr. Mary Gospodarowicz, President of the Union for International Cancer Control (UICC), will be one of the leadoffs of this year's meetings and present the esteemed Harvey Lecture. Also proffering invited presentations will be Drs. Vic Strecher (University of Michigan), Dr. Anthony Black (Seattle Cancer Coalition), and Dr. Bill Thompson (Fred Hutchinson Cancer Center). These presentations will certainly stimulate both the left and right sides of our brain. This year's meeting will also offer an exciting array of presentations in a series of concurrent sessions. On the Wednesday morning, there will be several workshops in the areas of Art-Based Cancer Education (Dr. Cueva), Responsible Conduct of Research (Dr. Chang), and Health Literacy (Dr. Payne), three timely and inspiring workshops led by three eminently qualified individuals. What a great way to start our meetings. A note to all presenters-I urge you all to turn your presentations into publications!

A review of the agenda for this year's meeting not only highlights where we are, but is also evidence of how far we've come. When the JCE began, a number of articles focused on the

A. M. Michalek

D'Youville College, Buffalo, NY, USA

A. M. Michalek $(\square)$

Roswell Park Cancer Institute, Buffalo, NY, USA

e-mail: arthur.michalek@roswellpark.org fatalistic attitudes of both cancer patients and the general public. Back then, it was a challenge to get people to say the word "cancer"; today we talk of survivorship. Today we discuss implementation of public programs focused on primary and secondary prevention: new models of interprofessional education, programs in tobacco cessation and nutrition, strategies related to patient communication, etc. We have made vast strides in how we relate to each other and to the general public and cancer patients. However, this is not the case everywhere in the world.

I had the privilege of participating in a cancer control workshop in Lagos, Nigeria, earlier this year. It was led by Dr. Chumy Nwogu, a colleague of mine at the Roswell Park Cancer Institute, and sponsored by the UICC. The broad goals of this workshop were to explore cancer prevention strategies and identify methods to improve cancer data collection and strategies to promote cost-effective collaborative cancer care. I believe that we were fairly successful in meeting these goals; however, we have a long way to go. Cancer incidence rates are increasing in developing countries due to increased exposure to cancer risk factors, reduced competition from infectious diseases, and increased life expectancy. In many ways, the state of cancer education in developing countries is similar to what we in the Americas faced in the 1950s and early 1960s. The public has a very negative and fatalistic view of cancer. This is not totally undeserved. Many nations lack the infrastructure and resources to effectively combat cancer. Facilities are ill equipped, and there are far too few professionals to deal with the mounting number of cancer cases. So what do we do... concede defeat or learn from past experience? Yes, it is time to go back to the future.

The 1985 blockbuster movie, "Back to the Future," tells the story of a young man who travels back in time where he accidentally meets and attracts the interest of his future mother [1]. This causes a schism in time affecting his parents' marriage and consequently his own existence. He must, therefore, travel back in time to repair the damage done. We are faced with a similar situation in the development and implementation of cancer 
control programs in developing countries. Cancer control in these nations lags behind programs in the Americas by 20 or 30 years. Cancer incidence rates are increasing, case fatality and mortality rates are high, most cancers are diagnosed in the late stage, cigarette smoking is either highly prevalent or on target to be so, diets are transforming from traditional to high-fat, lowfiber westernized diets, and communications about cancer are far from ideal. To redress the situation, we need to go back to the future to identify those cancer control programs proven to be most effective and to tailor and translate them to the local environ of developing countries. This will not be as easy as merely adopting existing programs. It will require significant cultural adjustments. It is not easy to adopt change at the expense of tradition. This concept was masterfully presented by the great Nigerian author Chinua Achebe in his novel Things Fall Apart [2]. It tells the story of a man growing from boyhood to adulthood in 1850s Nigeria where he is faced with the challenges of maintaining his cultural beliefs amidst changing times. In the end, change came at too great a price for the main character. The story, in the context of cancer control programming, emphasizes our need to not only initiate effective programming, but to translate it into the local culture so that it is not viewed as alien but as an extension of current traditions. This is easier said than done. It will require not just a change in people's perceptions, but the development of an infrastructure to meet their needs. Many of the individuals in these societies are faced with far greater challenges than early detection. These individuals live on less than a dollar a day, reside in less than desirable living quarters, lack proper sanitation and access to freshwater, pay out of pocket for many health benefits that we take for granted, etc. How does one begin to convince these individuals that what little money they have should be spent on healthcare and early detection and not basic sustenance or their children's future? We as cancer educators must take a step back and reevaluate our own positions. We must learn to understand the cultures and lifestyles of those whom we hope to help. For those who work in the international arena, I would recommend the Pulitzer Prize-winning book by Katherine Boo, Behind the Beautiful Forever, a book of nonfiction whose prose would rival any of the classics in fiction [3]. This is a powerful look from within a community resident in the slums of Annawadi, India. What are their thoughts and dreams? How do they manage to navigate their daily lives under such extreme conditions? These are thoughts I had as we drove by similar communities in Nigeria. How do we affect change in these societies? That is the challenge and why we need to go back to the future.

\section{References}

1. Universal Pictures (1985) Back to the future.

2. Achebe C (1958) Things fall apart. Heinemann Press, London

3. Boo K (2012) Behind the beautiful forevers. Random House, New York 